\title{
Screening of Rhizobacteria for Plant Growth Promotion and Their Tolerance to Drought Stress
}

\author{
RAHAYU FITRIANI WANGSA PUTRIE ${ }^{1}$, ARIS TRI WAHYUDI ${ }^{1}$, ABDJAD ASIH \\ NAWANGSIH $^{2}$, AND EDI HUSEN ${ }^{3}$ \\ 'Department of Biology, Faculty of Mathematics and Natural Sciences, Jalan Agatis, Institut Pertanian Bogor, \\ Kampus Dramaga Bogor 16680, Indonesia; \\ ${ }^{2}$ Department of Plant Protections, Faculty of Agriculture, Institut Pertanian Bogor, Jalan Agatis \\ Kampus Dramaga Bogor 16680, Indonesia; \\ ${ }^{3}$ Soil Research Institute, BBSDLP, Jalan Tentara Pelajar, Bogor 16114, Indonesia
}

\begin{abstract}
Rhizobacteria have been known for their capability as plant growth promoter through some mechanisms, directly and indirectly. The purpose of this research to screen rhizobacteria of Bacillus spp. and Pseudomonas spp. for their drought tolerance as plant growth promoter of maize (Zea mays). Screening of rhizobacteria as growth promoter of 47 isolates of Bacillus sp. CR and 34 isolates of Pseudomonas sp. CRB resulted 24 and 9 isolates were able to stimulate the growth of maize sprouts, respectively. Further screening of those growth promoter of the rhizobacterial isolates to drought tolerance resulted 7 isolates of Bacillus sp. CR and 6 isolates of Pseudomonas sp. CRB that were able to grow on medium with osmotic pressure -1 and $-2 \mathrm{MPa}$, respectively. Potential rhizobacterial isolates of growth promoter and drought tolerance were tested for antagonist mechanisms which aims to determine ability to live together in one carrier medium if to be made formulation. Both non antagonist rhizobacterial isolates were evaluated for their potential in producing exopolysaccharide (EPS) revealing that CRB 19 and CR 90 exhibited the highest activity of EPS production up to $0.346 \mathrm{mg} \mathrm{mL}^{-1}$ on medium with $-2.0 \mathrm{MPa}$ and $0.107 \mathrm{mg} \mathrm{mL}^{-1}$ on medium with $-0.73 \mathrm{MPa}$, respectively. Based on 16S rRNA sequence analysis, it revealed CRB 19 and CR 90 had highest similarities to Pseudomonas aeruginosa strain B2 and Brevibacillus brevis B33, respectively. Those growth promoter and drought tolerant of Bacillus sp. CR and Pseudomonas sp. CRB had potency to be developed as inoculants in dry land agriculture.
\end{abstract}

Key words: Bacillus sp. CR, drought tolerant, growth promoter, Pseudomonas sp. CRB, rhizobacteria

Rizobakteria diketahui memiliki kemampuan sebagai pemacu tumbuh tanaman melalui beberapa mekanisme, baik secara langsung maupun tidak langsung. Penelitian ini bertujuan untuk menyeleksi rizobakteria Bacillus spp. dan Pseudomonas spp. toleran kekeringan sebagai pemacu pertumbuhan tanaman jagung (Zea mays). Penapisan rizobakteria pemacu tumbuh dari 47 isolat Bacillus sp. CR dan 34 isolat Pseudomonas sp. CRB mendapatkan masing-masing 24 dan 9 isolat yang secara signifikan memacu pertumbuhan. Isolat pemacu tumbuh selanjutnya diseleksi terhadap toleran kekeringan, lalu didapatkan 7 isolat Bacillus sp. CR dan 6 isolat Pseudomonas sp. CRB yang dinyatakan toleran kekeringan. Masing-masing isolat mampu tumbuh pada media dengan tekanan osmotik -1 dan -2 MPa. Isolat potensial pemacu tumbuh toleran kekeringan diuji sifat antagonisnya untuk mengetahui kemampuannya hidup bersama pada satu media pembawa jika dibuat formula. Isolat yang tidak saling antagonis selanjutnya dievaluasi potensinya dalam menghasilkan eksopolisakarida. CRB 19 dan CR 90 masing-masing menghasilkan eksopolisakarida tertinggi hingga $0.346 \mathrm{mg} \mathrm{mL}^{-1}$ pada medium -2.0 MPa dan $0.107 \mathrm{mg} \mathrm{mL}^{-1}$ pada medium -0.73 MPa. Berdasarkan sekuens gen 16S rRNA, CRB 19 memiliki kemiripan tertinggi dengan Pseudomonas aeruginosa strain B2 dan CR 90 dengan Brevibacillus brevis B33. Bacillus sp. CR dan Pseudomonas sp. CRB pemacu tumbuh dan toleran kekeringan tersebut berpotensi untuk dikembangkan sebagai inokulan di lahan kering.

Kata kunci : Bacillus sp. CR, pemacu tumbuh, Pseudomonas sp. CRB, rizobakteria, toleran kekeringan

Rhizospheres are containing microorganisms that could increase plant growth. These bacteria are call plant growth promoting rhizobacteria (PGPR) that have a significant effect for plant growth, directly and indirectly. PGPR have been capability in producing indole acetic acid (IAA) hormone, exopolysaccharide (EPS), ACC-deaminase, as phosphate solubilizing,

*Corresponding author; Phone/Fax: 0251-8622833 , Email: aristri2011@gmail.com and biocontrol agents of pathogenic fungi (Dey et al. 2004; Kaci et al. 2005; Husen et al. 2011). IAA hormones is one of the plant growth hormone that plays a role in several aspects of plant growth, including cell division and elongation, differentiation, tropisms, apical dominance, aging, abscission, and flowering (Zhao et al. 2001).

Drought can affect microorganisms and plant growth since water becomes a limiting factor for plants 
and microorganisms to survive. One way drought adaptation of microorganisms are secretion of exopolysaccharide (EPS) in higher amounts. EPS are structural component of extracellular matrix in biofilms are synthesized by cells to respond physiological stress in the environment (Marvasi et al. 2010). EPS were produced as response biotic and abiotic stress factors to adaptation in extreme environments. The main function is to assist the protection against environmental stresses. Microorganisms such as Agrobacterium sp., Alcaligenes faecalis, Xanthomonas campestris, Bacillus sp., Zygomonas mobilis, Leuconostoc, Pseudomonas sp., Acetobacter xylinum, and several other genera of microorganisms are known to produce EPS (Donot et al. 2011). EPS production by Rhizobium sullae KYGT207 strain was isolated from dry land in South Algeria (Gassi Touil) and was known to contribute of water absorption and nutrients by roots through modification of physical properties of Triticum durum rhizosphere. In vivo test which inoculated KYGT207 strain on wheat plants was also known to give significant influence as the plant growth promotion. EPS in sandy soil can protect plants from stress, lack of water and contributes to the formation of soil aggregates (Kaci et al. 2005). Previously we have isolated two groups of PGPR, Bacillus sp. CR and Pseudomonas sp. CRB from rhizosphere of soybean plant of Cirebon field area West Java (Wahyudi et al. 2011 a.b). The use of drought tolerance of PGPR in plant growth promotion is an effective and environmentally friendly step. Objective of this study was to screen PGPR Pseudomonas sp. CRB and Bacillus sp. CR for plant growth promoting of maize under drought stress condition.

\section{MATERIALS AND METHODS}

Screening of Pseudomonas sp. and Bacillus sp. as Growth Promoter of Maize. Initial stage was seed surface sterilization (Somasegaran and Hoben 1985). Sterilized seeds were then soaked in distilled water for 24 $\mathrm{h}$ to speed up the germination process. After $24 \mathrm{~h}$, seeds were placed in Petri dishes covered by filter paper that was previously moistened with distilled water for $24 \mathrm{~h}$. Fourty seven isolates of Bacillus sp. CR and 34 isolates of Pseudomonas sp. CRB were cultured on nutrient broth (NB) (yeast extract $2 \mathrm{~g} \mathrm{~L}^{-1}$, peptone $5 \mathrm{gL}^{-1}, \mathrm{NaCl} 5$ $\mathrm{g} \mathrm{L}^{-1}$ ) and King's B medium (peptone $20 \mathrm{~g} \mathrm{~L}^{-1}, \mathrm{~K}_{2} \mathrm{HPO}_{4}$ $1.5 \mathrm{~g} \mathrm{~L}^{-1}, \mathrm{MgSO}_{4} 1.5 \mathrm{~g} \mathrm{~L}^{-1}$, and glycerol $15 \mathrm{~mL} \mathrm{~L}^{-1}$ ) shaked at $120 \mathrm{rpm}$ for $24 \mathrm{~h}$ at room temperature, respectively. Seeds that have been germinated with $2-3 \mathrm{~mm}$ long of primary roots were transferred to a Petri dish that contains $1 \%$ water agar medium and each seed was inoculated with $100 \mu \mathrm{L}$ culture of Bacillus sp. CR or Pseudomonas sp. CRB with a cell density of $10^{9}$ cells $\mathrm{mL}^{-1}$. Seeds with medium without inoculum were used as a control. Seeds that had been inoculated with bacteria were incubated for $7 \mathrm{~d}$ at room temperature in dark conditions. Growth parameters measured were length of stem, root length, and number of lateral roots (Dey et al. 2004). Data obtained from this experiments were statistically analyzed by one-way Analysis of Variance (ANOVA) using SAS 9.1 software and followed by Duncan's test (DMRT) at $\%$ level.

Screening of Drought Tolerance. Bacillus sp. CR and Pseudomonas sp. CRB were cultured in nutrient broth (NB) medium. Two hundred $\mu \mathrm{L}$ preculture of each isolates was inoculated in $20 \mathrm{~mL}$ of medium containing polyethylene glycol 6000 (PEG 6000) with concentrations of $0,-0.73,-1,-1.5,-2$, and $-2.5 \mathrm{MPa}$. Concentration of osmotic pressure arrangements according to PEG 6000 calculated by Michel and Kauffman (1973). Incubation of medium that had been inoculated with bacterial isolate was performed at room temperature for $24 \mathrm{~h}$ by shaking at $120 \mathrm{rpm}$. Optical density (OD) of each bacterial culture was measured using spectrophptometer at a wavelength of $570 \mathrm{~nm}$. Medium without bacterial inoculation was used as a control. Bacteria that were able to grow minimum at $-0.73 \mathrm{MPa}$ with OD 0.4 catagorized as drought tolerant (Sandhya et al. 2009).

Antagonism Test. The purpose of this test was to determine capability of Bacillus sp. CR and Pseudomonas sp. CRB to live together in one medium with no competition each other if to be made formulated. Each isolates was grown in LB medium (tryptone $10 \mathrm{~g} \mathrm{~L}^{-1}, \mathrm{NaCl} 10 \mathrm{~g} \mathrm{~L}^{-1}$, and yeast extract $5 \mathrm{~g} \mathrm{~L}^{-}$ $\left.{ }^{1}\right)$ at $120 \mathrm{rpm}$ for $24 \mathrm{~h}$ to reach concentration of $10^{8}$ cells $\mathrm{mL}^{-1}$. Antagonism test was done by the following ways: $10 \mathrm{~mL}$ of LA medium was inoculated with $100 \mu \mathrm{L}$ targets culture, then poured into a sterile Petri dish. A total of $1 \mathrm{~mL}$ test culture on LB medium with cell density of $10^{8}$ cells $\mathrm{mL}^{-1}$ was centrifuged $10 \mathrm{~min}$ at 10 $000 \mathrm{rpm}$ to obtain the supernatant futher used to antagonism test. Sterile paper discs were given for each supernatant test culture then placed on the dish containing LA medium that had been inoculated with the targets culture for further incubated. Each isolate $\mathrm{CR}$ and $\mathrm{CRB}$ were used as the test culture and targets culture. Antagonism was shown by a clear zone around the paper disc.

Test of Exopolysaccharide (EPS) Production. 
Isolates of Bacillus sp. CR and Pseudomonas sp. CRB were grown in NB medium added by PEG 6000 to induction of drought stress. Culture incubation was performed in an incubator shaker at room temperature for $72 \mathrm{~h}$, furthermore the cultures were centrifuged to separate the supernatant and pellet. Each supernatant was mixed with $3 \mathrm{~mL}$ of cold absolute alcohol and incubated overnight at $4{ }^{\circ} \mathrm{C}$. EPS was obtained by centrifugation for $15 \mathrm{~min}$ at $10000 \mathrm{rpm}$. Quantity of total carbohydrate content which settles on the EPS was measured using the method described by Dubois et al (1956). Optical density of each EPS sample was measured using spectrophotometer at a wavelength of $490 \mathrm{~nm}$.

Phospate Solubilization Test. Test of phosphate solubilization was performed by standard methods. Pseudomonas sp. CRB or Bacillus sp. CR was grown by streaking on Pikovskaya Agar plate medium containing tricalcium phosphate (glucose $10 \mathrm{~g} \mathrm{~L}^{-1}$, $\mathrm{NaCl} 0.2 \mathrm{~g} \mathrm{~L}^{-1}, \mathrm{KCl} 0.2 \mathrm{~g} \mathrm{~L}^{-1}, \mathrm{MgSO}_{4} \cdot 7 \mathrm{H}_{2} \mathrm{O} 0.1 \mathrm{~g} \mathrm{~L}^{-1}$, $\mathrm{MnSO}_{4} \cdot 2 \mathrm{H}_{2} \mathrm{O} 2.5 \mathrm{mg} \mathrm{L}^{-1}, \mathrm{FeSO}_{4} .7 \mathrm{H}_{2} \mathrm{O} 2.5 \mathrm{mg} \mathrm{L}^{-1}$, yeast extract $\mathrm{g} \mathrm{L}^{-1},\left(\mathrm{NH}_{4}\right)_{2} \mathrm{SO}_{4} 1 \mathrm{gL}-1$, and $\left.\mathrm{Ca}_{3}\left(\mathrm{PO}_{4}\right)_{2} 5 \mathrm{~g} \mathrm{~L}^{-1}\right)$. Plates were incubated at room temperature for $72 \mathrm{~h}$. Clear zone formed around the colony indicated the bacteria solubilized phosphate. The clear zone was formed by these bacteria were measured to determine phosphate solubilization index (PSI).

$$
\underset{\text { solubilizing index }}{\text { Phosphate }}=\frac{\text { clear zone diameter - colony }}{\text { colony diameter }}
$$

\section{Molecular Identification Based on 16S rRNA}

Sequence. Bacterial genomes were extracted by the Cetyl Trimetyl Ammonium Bromide (CTAB) method. 16S rRNA gene was amplified by PCR with 63F primers (5'-CAGGCCTAACACATGCAAGTC-3') and 1387R (5'-GGGCGGWTGGTACAAGGC-3') (Marchesi et al. 1998) in total volume of $50 \mu \mathrm{L}$. The PCR volume contains of $1 \mu \mathrm{L}$ of genomic DNA template; $1 \mu \mathrm{L}$ of primer for each forward and reverse, $25 \mu \mathrm{L}$ of $2 \mathrm{X}$ Phusion Master Mix (Thermo Scientific Phusion High Fidelity), $1 \mu \mathrm{L}$ of DMSO, and $\mathrm{dd}_{2} \mathrm{O}$ to a volume of $50 \mu \mathrm{L}$. Amplification was performed for 30 cycles that include stages of initial denaturation at a temperature of $94{ }^{\circ} \mathrm{C}$ for $2 \mathrm{~min}$, denaturation at a temperature of $92{ }^{\circ} \mathrm{C}$ for $30 \mathrm{sec}$, annealing at a temperature of $55^{\circ} \mathrm{C}$ for $30 \mathrm{sec}$, extension at $72^{\circ} \mathrm{C}$ for 1 min, and final extension at $72{ }^{\circ} \mathrm{C}$ temperature for $7 \mathrm{~min}$. DNA of PCR products were purified and sequenced and analyzed by comparing the sequences with GenBank database using the BlastN program to determine similarity. The DNA sequences were also analyzed their phylogenetic tree using Mega5 and ClustalW software.

\section{RESULTS}

Results of this study, 24 out of 47 isolates of Bacillus sp. CR and 9 out of 34 isolates of Pseudomonas sp. CRB screened for growth promotion revealed that they had capability in promoting growth of maize sprouts. Bacillus could increase the growth of shoot length, root length, and number of lateral root of maize sprouts. Pseudomonas could increase the growth of root length and stem length of maize sprouts. This results also revealed that Bacillus sp. CR 36 was able to increase all parameters of the growth of maize, significantly (Table 1,2).

All of the rhizobacteria selected as plant growth promoting were screened for their drought tolerance. Seven isolates of Bacillus sp. CR and six isolates of Pseudomonas sp. CRB were classified as drought tolerant, they were able to grow in medium with -1.0 $\mathrm{MPa}$ for Bacillus sp. CR, and $-2.0 \mathrm{MPa}$ for Pseudomonas sp. CRB (Table 3 and 4). Rhizobacteria Pseudomonas sp. CRB, and Bacillus sp. CR which could grow in the highest osmotic presssure were subjected to antagonism test to know their capability to survive in one medium. Results of this experiment are performed in Table 5 and Table 6. Moreover, the potent isolate of rhizobacteria, $\mathrm{CRB}$ and $\mathrm{CR}$ were examined for their EPS production. Isolate CRB 19 exhibited the highest activity of EPS production to $0.346 \mathrm{mg} \mathrm{mL}^{-1}$ in NB medium with $-2.0 \mathrm{MPa}$ of osmotic pressure. Average of EPS produced by Pseudomonas sp. CRB was higher than Bacillus sp. CR. Bacillus sp. CR produced EPS within a range of 0.050-0.107 $\mathrm{mg} \mathrm{mL}^{-1}$ on medium with $-0.73 \mathrm{MPa}$ of osmotic pressure and $0.072-0.096 \mathrm{mg} \mathrm{mL}^{-1}$ on medium with -1 MPa of osmotic pressure (Fig 1A). Pseudomonas sp. CRB was able to produce EPS within a range of $0.062-0.196 \mathrm{mg} \mathrm{mL}^{-1}$ on medium with $-0.73 \mathrm{MPa}$ of osmotic pressure and within a range 0.055-0.197 mg mL $\mathrm{mL}^{-1}$ on medium with $-1 \mathrm{MPa}$ of osmotic pressure (Fig 1B). Majority, Bacillus sp. CR and Pseudomonas sp. CRB have been known their ability as phospate solubilizing on Pikovskaya agar medium. Pseudomonas sp. CRB 10 was known as the highest in phospate solubilizing. Phosphate solubilizing index of Pseudomonas sp. CRB and Bacillus sp. CR within a range of 0.18-0.67. All those isolates of rhizobacteria were able to solubilyze 
Table 1 Effect of Bacillus sp. isolates in some growth parameters of maize

\begin{tabular}{|c|c|c|c|}
\hline Treatment & Shoot length $(\mathrm{cm})$ & Root length $(\mathrm{cm})$ & Number of lateral root \\
\hline CR 61 & $15.5 \mathrm{a}$ & $13.8_{\mathrm{n}}$ & $14.0_{\text {lmnopqr }}$ \\
\hline CR 39 & $15.0_{a b}$ & $15.0_{\mathrm{lnm}}$ & $24.4_{c}$ \\
\hline CR 6 & 14.6 abc & $16.8_{\mathrm{ijkl}}$ & $24.8_{\mathrm{c}}$ \\
\hline CR 59 & 14.5 abcd & $19.7 \mathrm{defgh}$ & $12.6 \mathrm{pqr}$ \\
\hline CR 32 & 14.3 abcd & $17.4_{\text {hijk }}$ & 14.8 klmnopq \\
\hline CR 69 & 14.2 abcd & $25.2_{b}$ & $20.2 \mathrm{de}$ \\
\hline CR 42 & 13.9 abcde & $15.1_{\mathrm{lnm}}$ & $11.0_{\mathrm{r}}$ \\
\hline CR 12 & 13.7 abcdef & $22.5_{c}$ & 19.6 defg \\
\hline CR 36 & 13.5 abcdefg & $25.5_{b}$ & $28.0_{\mathrm{ab}}$ \\
\hline CR 3 & 13.3 abcdefgh & $25.7_{b}$ & $12.2 \mathrm{pqr}$ \\
\hline CR 51 & $13.1_{\text {abcdefgh }}$ & $21.1_{\text {cdef }}$ & 19.4 defgh \\
\hline CR 81 & 12.9 bcdefghij & $20.8_{\text {cdef }}$ & 13.6 mnopqr \\
\hline CR 79 & $12.8_{\text {bcdefghij }}$ & 29.6 a & $26.0_{\mathrm{bc}}$ \\
\hline CR 30 & 12.5 bcdefghijk & $17.7_{\text {hijk }}$ & 13.4 nopqr \\
\hline CR 83 & $12.4_{\text {bcdefghijk }}$ & $28.8_{a}$ & 17.8 efghijk \\
\hline CR 22 & $12.4_{\text {bcdefghijk }}$ & $22.3_{\mathrm{c}}$ & 13.8 mnopqr \\
\hline CR 86 & 12.3 bcdefghijk & $14.4 \mathrm{mn}$ & 29.8 a \\
\hline CR 31 & 12.2 cdefghijk & $25.2_{b}$ & $21.4_{\mathrm{d}}$ \\
\hline CR 74 & 12.2 cdefghijk & $22.5 \mathrm{c}$ & 16.4 ghijklmn \\
\hline CR 54 & 12.1 cdefghijk & $18.4_{\text {ghij }}$ & 16.2 hijklmn \\
\hline CR 2 & $12.1_{\text {cdefghijk }}$ & $25.1_{b}$ & $19.4_{\text {defgh }}$ \\
\hline CR 56 & $12.1_{\text {cdefghijk }}$ & $18.8_{\mathrm{fgh}} \mathrm{i}$ & 13.2 nopqr \\
\hline CR 66 & 12.0 cdefghijk & $21.7 \mathrm{~cd}$ & 16.2 hijklmn \\
\hline CR 25 & $12.0_{\text {cdefghijk }}$ & $21.9 \mathrm{~cd}$ & 15.2 jklmnop \\
\hline CR 90 & 11.9 cdefghijk & $25.5_{b}$ & 17.6 efghijk \\
\hline CR 21 & 11.9 cdefghijk & $21.8 \mathrm{~cd}$ & $24.4_{c}$ \\
\hline CR 88 & $11.8_{\text {defghijk }}$ & $16.2 \mathrm{jklm}$ & 17.2 efghijkl \\
\hline CR 34 & $11.8_{\text {defghijk }}$ & $17.0_{\mathrm{ijkl}}$ & $26.6_{b c}$ \\
\hline CR 15 & 11.7 defghijk & $19.1 \mathrm{efghi}$ & $11.8 \mathrm{qr}$ \\
\hline CR 29 & 11.4 efghijk & $20.8_{\text {cdef }}$ & 19.0 defghi \\
\hline CR 26 & $11.4_{\text {efghijk }}$ & $22.2 \mathrm{~cd}$ & 15.0 jklmnopq \\
\hline CR 17 & 11.2 efghijk & 20.7 cdefg & $12.6 \mathrm{pqr}$ \\
\hline CR 64 & $11.1_{\text {fghijk }}$ & $21.5 \mathrm{~cd}$ & 18.2 efghij \\
\hline CR 50 & $11.0_{\text {fghijk }}$ & $15.7 \mathrm{klmn}$ & 12.8 орqr \\
\hline CR 27 & $10.9_{\text {ghijk }}$ & $25.1_{b}$ & $11.2_{\mathrm{r}}$ \\
\hline CR 13 & $10.7_{\text {ghijk }}$ & $14.2 \mathrm{~nm}$ & $20.0_{\text {def }}$ \\
\hline CR 91 & $10.7_{\text {ghijk }}$ & $22.7 \mathrm{c}$ & 14.0 Imnopqr \\
\hline CR 23 & $10.7_{\text {ghijk }}$ & $17.0_{\mathrm{ijkl}}$ & 18.0 efghij \\
\hline CR 75 & $10.7_{\text {ghijk }}$ & $21.4_{\mathrm{cde}}$ & $18.6_{\text {defghi }}$ \\
\hline CR 55 & $10.6_{\text {hijk }}$ & $22.2_{\mathrm{c}}$ & 16.0 ijklmno \\
\hline CR 8 & 10.6 hijk & $25.2_{\mathrm{b}}$ & $12.6 \mathrm{pqr}$ \\
\hline CR 67 & $10.4_{\mathrm{ijk}}$ & $22.1 \mathrm{~cd}$ & $24.6_{c}$ \\
\hline Control & $10.3_{\mathrm{ijk}}$ & 20.2 cdefg & $17.4_{\text {efghijk }}$ \\
\hline CR 38 & $10.2 \mathrm{jk}$ & $18.8_{\text {fghi }}$ & $19.0_{\text {defghi }}$ \\
\hline CR 24 & $10.2 \mathrm{jk}$ & $22.2 \mathrm{~cd}$ & 14.0 Imnopqr \\
\hline CR 46 & $10.1_{\mathrm{jk}}$ & $25.0_{b}$ & $24.6_{c}$ \\
\hline CR 33 & $10.0_{\mathrm{k}}$ & 27.9 a & $13.6_{\text {mnopqr }}$ \\
\hline CR 47 & $9.70_{\mathrm{k}}$ & $21.7 \mathrm{~cd}$ & 16.8 fghijklm \\
\hline
\end{tabular}

Numbers within a column followed by the same letter are not significantly different at $5 \%$ level by DMRT $(\alpha=0.05)$. Bold indicated growth promoter isolates compared with control.

phosphate, except CR 67, CR 90, and CRB 4.

Futhermore, eight potential isolates of rhizobacteria
Pseudomonas sp. CRB and Bacillus sp. CR classified as growth promoter of maize and drought tolerant, were 
Table 2 Effect of Pseudomonas sp. isolates in some growth parameters of maize

\begin{tabular}{|c|c|c|c|c|c|c|c|c|}
\hline Treatment & \multicolumn{2}{|c|}{$\begin{array}{l}\text { Root length } \\
(\mathrm{cm})\end{array}$} & \multicolumn{2}{|c|}{$\begin{array}{l}\text { Shoot length } \\
(\mathrm{cm})\end{array}$} & \multirow{2}{*}{$\begin{array}{c}\text { Treatment } \\
\text { Group } 4\end{array}$} & \multirow[t]{2}{*}{$\begin{array}{l}\text { Root length } \\
\quad(\mathrm{cm})\end{array}$} & \multicolumn{2}{|c|}{$\begin{array}{l}\text { Shoot length } \\
(\mathrm{cm})\end{array}$} \\
\hline Group 1 & & & & & & & & \\
\hline Control & 10.8 & $a b c$ & 17.5 & $a b c$ & Control & $6.6 \mathrm{abc}$ & 12.7 & abcd \\
\hline CRB 42 & 9.6 & $a b c$ & 12.1 & $a b c$ & CRB 64 & $9.2 \mathrm{cdef}$ & 18.9 & d \\
\hline CRB 55 & 10.1 & $a b c$ & 13.1 & $a b c$ & CRB 85 & $6.1 \mathrm{ab}$ & 12.8 & abcd \\
\hline CRB 107 & 11.7 & $a b c$ & 15.4 & $a b c$ & CRB 71 & 10.2 ef & 18.8 & $d$ \\
\hline CRB 88 & 11.4 & $a b c$ & 19.4 & $a b c$ & CRB 3 & $9.3 \mathrm{cdef}$ & 18.0 & $\mathrm{~cd}$ \\
\hline Group 2 & & & & & CRB 4 & $10.6 \mathrm{f}$ & 15.0 & abcd \\
\hline Control & 10.9 & $a b$ & 10.1 & $\mathrm{a}$ & CRB 112 & 8.9 cdef & 19.1 & d \\
\hline CRB 34 & 12.4 & $a b c$ & 16.2 & $\mathbf{b}$ & CRB 90 & 8.7 bcdef & 13.8 & abcd \\
\hline CRB 111 & 11.9 & $a b$ & 14.4 & $a b$ & CRB 25 & 7.5 abcde & 11.2 & $a b$ \\
\hline CRB 19 & 14.2 & bcd & 16.9 & $\mathbf{b}$ & CRB 104 & $5.7 \mathrm{a}$ & 10.4 & $\mathrm{a}$ \\
\hline CRB 24 & 18.7 & d & 15.6 & $\mathbf{b}$ & CRB 76 & 8.0 abcdef & 13.9 & abcd \\
\hline CRB 115 & 7.9 & $\mathrm{a}$ & 13.1 & $a b$ & CRB 113 & 8.7 bcdef & 13.4 & abcd \\
\hline CRB 47 & 17.8 & cd & 14.7 & b & CRB 96 & 7.6 abcde & 12.9 & abcd \\
\hline CRB 77 & 9.3 & $a b$ & 10.1 & $\mathrm{a}$ & CRB 33 & 8.7 bcdef & 12.0 & $a b c$ \\
\hline CRB 58 & 10.4 & $a b$ & 12.6 & $a b$ & CRB 36 & 8.1 abcdef & 15.7 & abcd \\
\hline CRB 10 & 12.3 & $a b c$ & 16.9 & $\mathbf{b}$ & CRB 69 & 8.8 bcdef & 17.4 & bcd \\
\hline Group 3 & & & & & CRB 63 & 8.2 abcdef & 16.9 & bcd \\
\hline Control & 11.3 & $\mathrm{a}$ & 10.2 & $\mathrm{a}$ & CRB 40 & 9.1 cdef & 17.9 & $\mathrm{~cd}$ \\
\hline CRB 23 & 14.0 & $\mathrm{a}$ & 16.6 & $\mathbf{b}$ & CRB 92 & 7.1 abcd & 18.6 & $\mathrm{~cd}$ \\
\hline CRB 98 & 13.0 & $\mathrm{a}$ & 16.5 & b & & & & \\
\hline CRB 100 & 9.6 & $\mathrm{a}$ & 9.8 & $\mathrm{a}$ & & & & \\
\hline
\end{tabular}

Numbers within a column followed by the same letter are not significantly different at $5 \%$ level by. DMRT $(\alpha=0.05)$. Bold indicated growth promoter isolates compared with control.

Table 3 Optical density values of Bacillus sp. isolates in osmotic pressure of $0,-0.73,-1$, and $-1.5 \mathrm{Mpa}$ at $\lambda 570 \mathrm{~nm}$

\begin{tabular}{ccccc}
\hline $\begin{array}{c}\text { Isolates } \\
\text { code }\end{array}$ & \multicolumn{4}{c}{ Osmotic pressure } \\
\cline { 2 - 5 } $\mathbf{0 ~ M P a}$ & $\mathbf{- 0 . 7 3 ~ M P a ~}$ & $\mathbf{- 1 ~ M P a ~}$ & $\mathbf{- 1 . 5 ~ M P a ~}$ \\
\hline CR 33 & $\mathbf{1 . 5 1 4}$ & $\mathbf{0 . 5 0 4}$ & $\mathbf{0 . 4 0 0}$ & 0.071 \\
CR 61 & 1.273 & 0.400 & 0.290 & 0.000 \\
CR 69 & 1.011 & 0.681 & 0.271 & 0.000 \\
CR 36 & $\mathbf{1 . 4 2 5}$ & $\mathbf{0 . 4 7 1}$ & $\mathbf{0 . 4 1 2}$ & 0.064 \\
CR 83 & $\mathbf{1 . 0 7 3}$ & $\mathbf{0 . 7 5 8}$ & $\mathbf{0 . 4 6 4}$ & 0.017 \\
CR 39 & $\mathbf{1 . 1 1 8}$ & $\mathbf{0 . 7 6 3}$ & $\mathbf{0 . 4 8 3}$ & 0.056 \\
CR 51 & 1.317 & 0.496 & 0.138 & 0.000 \\
CR 46 & $\mathbf{1 . 0 5 6}$ & $\mathbf{0 . 5 4 7}$ & $\mathbf{0 . 4 7 6}$ & 0.022 \\
CR 67 & $\mathbf{0 . 9 5}$ & $\mathbf{0 . 5 0 3}$ & $\mathbf{0 . 4 3 2}$ & 0.033 \\
CR 31 & 1.069 & 0.698 & 0.329 & 0.000 \\
CR 90 & $\mathbf{1 . 0 6 3}$ & $\mathbf{0 . 6 8 2}$ & $\mathbf{0 . 5 5 9}$ & 0.028 \\
CR 32 & 1.11 & 0.763 & 0.326 & 0.000 \\
\hline
\end{tabular}

Bold of figures and letters indicated drought tolerant isolates. MPa: Mega Pascal

molecularly indentified based on 16S rRNA gene and sequence (Table 7 and 8). Phylogenetic tree analysis using neighboor joining method (Fig $2 \mathrm{~A}, \mathrm{~B}$ ).

\section{DISCUSSION}

Some studies suggest that Bacillus sp. and 
Table 4 OD values of Pseudomonas sp. isolates in osmotic pressure of $0 \mathrm{MPa}$ to $-2.5 \mathrm{MPa}$ at $\lambda 570 \mathrm{~nm}$

\begin{tabular}{lcccccc}
\hline Isolates Code & \multicolumn{5}{c}{ Osmotic pressure } \\
\cline { 2 - 7 } & $\mathbf{0 ~ M P a}$ & $\mathbf{- 0 . 7 3 ~ M P a ~}$ & $\mathbf{- 1} \mathbf{~ M P a}$ & $\mathbf{- 1 . 5} \mathbf{~ M P a}$ & $\mathbf{- 2} \mathbf{~ M P a}$ & $\mathbf{- 2 . 5} \mathbf{~ M P a}$ \\
\hline CRB 4 & 1.742 & 1.362 & 1.254 & 0.966 & 0.792 & 0.490 \\
CRB 10 & 1.084 & 0.773 & 0.727 & 0.843 & 0.725 & 0.173 \\
CRB 19 & 1.789 & 1.058 & 1.017 & 1.086 & 0.872 & 0.563 \\
CRB 23 & 1.719 & 1.654 & 1.331 & 0.506 & 0.431 & 0.000 \\
CRB 47 & 1.709 & 1.249 & 1.151 & 0.771 & 0.630 & 0.000 \\
CRB 98 & 1.713 & 1.092 & 1.042 & 1.015 & 0.831 & 0.664 \\
\hline
\end{tabular}

OD value $\geq 0.4$ indicated drought tolerant isolates. MPa: Mega Pascal

Table 5 Inhibition zone formation by Bacillus sp. (CR) as target bacteria against Pseudomonas sp. (CRB) as test bacteria

\begin{tabular}{lccccccc}
\hline $\begin{array}{c}\text { Target } \\
\text { Isolates }\end{array}$ & \multicolumn{7}{c}{ Test isolates } \\
\cline { 2 - 7 } & CR 33 & CR 39 & CR 83 & CR 67 & CR 90 & CR 36 & CR 46 \\
\hline CRB 98 & + & + & - & - & + & + & - \\
CRB 23 & - & + & - & - & + & - & - \\
CRB 10 & - & - & - & - & - & + & - \\
CRB 47 & - & - & - & - & - & + & - \\
CRB 4 & - & - & - & - & - & + & - \\
CRB 19 & - & - & + & - & - & + & - \\
\hline
\end{tabular}

$\begin{array}{lll}\text { CR } & : \text { Bacillus sp. } & (+): \text { no clear zone } \\ \text { CRB } & : \text { Pseudomonas sp. } & (-): \text { no clear zone }\end{array}$

Table 6 Inhibition zone formation by Pseudomonas sp. (CRB) as target bacteria against Bacillus sp. (CR) as test bacteria

\begin{tabular}{lcccccc}
\hline \multirow{2}{*}{$\begin{array}{c}\text { Target } \\
\text { isolates }\end{array}$} & CRB 98 & CRB 23 & CRB 10 & CRB 47 & CRB 4 & CRB 19 \\
\cline { 2 - 7 } CR 33 & - & - & - & - & - & - \\
CR 39 & + & + & - & - & - & - \\
CR 83 & - & - & - & - & - & + \\
CR 67 & - & - & - & - & - & - \\
CR 90 & + & + & - & - & - & - \\
CR 36 & + & - & + & + & + & + \\
CR 46 & - & - & - & - & - & - \\
\hline
\end{tabular}

\begin{tabular}{ll}
\hline Information : & \\
CR $\quad:$ Bacillus sp. & $(+):$ no clear zone \\
CRB $\quad:$ Pseudomonas sp. & $(-):$ no clear zone
\end{tabular}

Pseudomonas sp. have been capability to increase plant height by producing IAA hormones (Patten and Glick 2002; Leveau and Lindow 2005; Wahyudi et al. 2011a,b). Rhizobacteria as growth promoter in this research previously also had been tested in producing IAA hormone within a range 2.82-22.79 ppm (Wahyudi et al. 2011a,b). Other isolates, 23 strains of Bacillus and 25 strains of Pseudomonas did not have ability as growth promotion of maize sprouts. This might be caused by their IAA concentration is too high, for example isolate CR 55 producing IAA up to 44.66 ppm (Wahyudi et al. 2011a). The highest of IAA concentration was known to stimulate the growth of maize using biological fertilizer within a range 54.55 ppm in leaves and $22.68 \mathrm{ppm}$ in roots, respectively. Leaf tissue contains a higher IAA concentration rather than root tissue (Wibowo 2008). High levels of IAA hormone would promote formation of ethylene and 

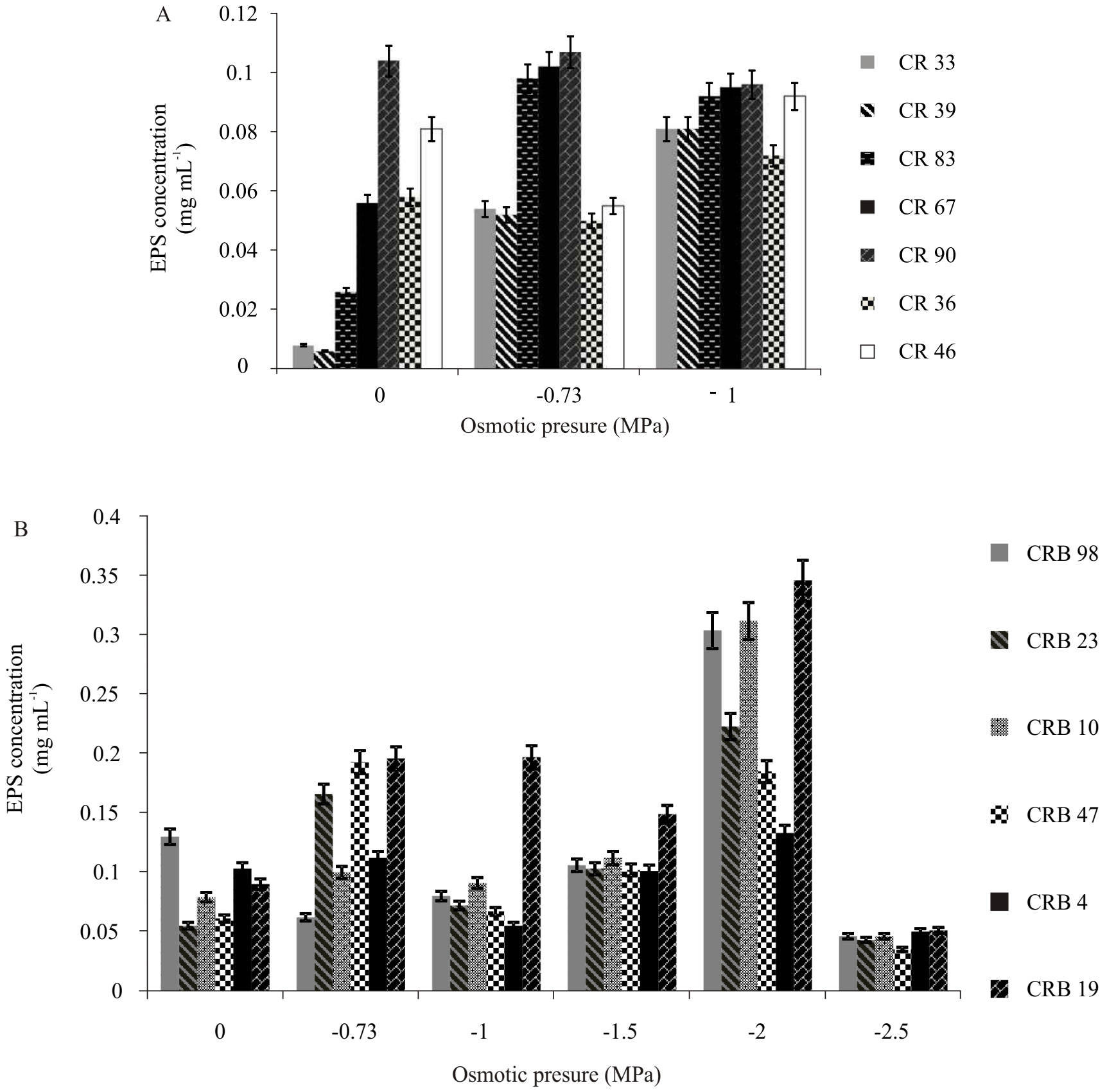

Fig 1 EPS concentration produced by Bacillus sp. CR (A) and Pseudomonas sp. CRB (B) at different osmotic pressure.

Table 7 Identification of 16S rRNA gene sequence homology of isolates of Bacillus sp. CR with sequences available in Genbank using BlastN program

\begin{tabular}{|c|c|c|c|c|c|c|}
\hline Isolates & $\begin{array}{l}\text { Species most } \\
\text { related }\end{array}$ & $\begin{array}{l}\text { Sequence } \\
\text { similarity }\end{array}$ & Length (bp) & $\begin{array}{l}\text { Query } \\
\text { Cover }\end{array}$ & E-Value & Accession number \\
\hline CR 46 & $\begin{array}{l}\text { Bacillus isabeliae } \\
\text { strain CVS-8 }\end{array}$ & $83 \%$ & $846 / 1018$ & $82 \%$ & 0.00 & NR 042619.1 \\
\hline CR 67 & $\begin{array}{l}\text { Brevibacillus brevis } \\
\text { strain NBRC } 15304\end{array}$ & $100 \%$ & $659 / 659$ & $100 \%$ & 0.00 & NR 041524.1 \\
\hline CR 83 & $\begin{array}{l}\text { Bacillus cereus } \\
\text { ATCC } 14579 \text { strain } \\
\text { ATCC } 14579\end{array}$ & $100 \%$ & $566 / 566$ & $100 \%$ & 0.00 & NR 074540.1 \\
\hline CR 90 & $\begin{array}{l}\text { Brevibacillus brevis } \\
\text { strain bB33 }\end{array}$ & $97 \%$ & $1200 / 1243$ & $92 \%$ & 0.00 & JF772474.1 \\
\hline
\end{tabular}


Table 8 Identification of 16S rRNA gene sequence homology isolates of Pseudomonas sp. CRB with sequences available in Genbank using BlastN program

\begin{tabular}{llccccc}
\hline Isolates & Species most related & $\begin{array}{l}\text { Sequence } \\
\text { similarity }\end{array}$ & Length (bp) & $\begin{array}{l}\text { Query } \\
\text { Cover }\end{array}$ & E-Value & Accession number \\
\hline CRB 10 & $\begin{array}{l}\text { Pseudomonas } \\
\text { aeruginosa } \text { strain L1 }\end{array}$ & $99 \%$ & $434 / 440$ & $94 \%$ & 0.00 & JX292018.1 \\
CRB 19 & $\begin{array}{l}\text { Pseudomonas } \\
\text { aeruginosa } \text { strain B2 }\end{array}$ & $91 \%$ & $966 / 1062$ & $98 \%$ & 0.00 & JQ900536.1 \\
CRB 23 & $\begin{array}{l}\text { Pseudomonas fragi } \text { strain } \\
\text { ATCC 4973 }\end{array}$ & $84 \%$ & $657 / 780$ & $83 \%$ & 0.00 & NR 024946.1 \\
CRB 98 & $\begin{array}{l}\text { Pseudomonas } \text { sp. CL 3.1 } \\
\text { CR }\end{array}$ & $97 \%$ & $1297 / 1334$ & $99 \%$ & 0.00 & FM173664.1 \\
\hline
\end{tabular}

A

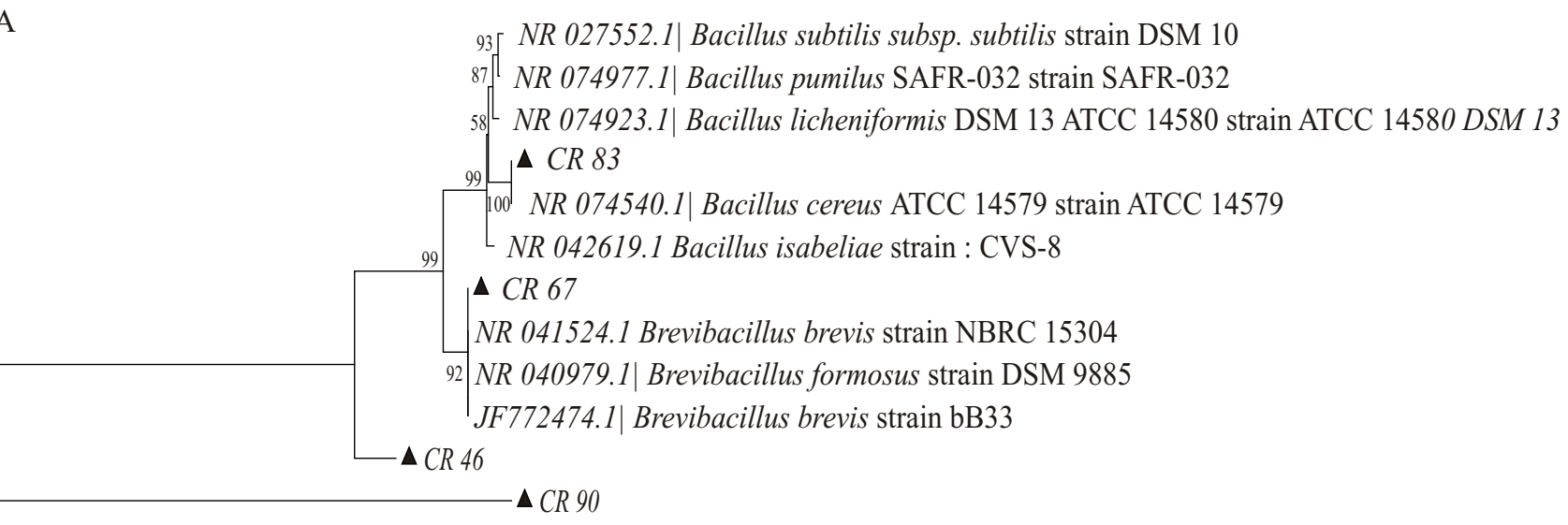

0.1

B

${ }_{93}^{77}\left[\begin{array}{l}\text { NR 024946.1| Pseudomonas fragi strain ATCC } 4973 \\ \text { NR 044974.1| Pseudomonas chlororaphis subsp. chlororaphis strain DSM 50083T }\end{array}\right.$

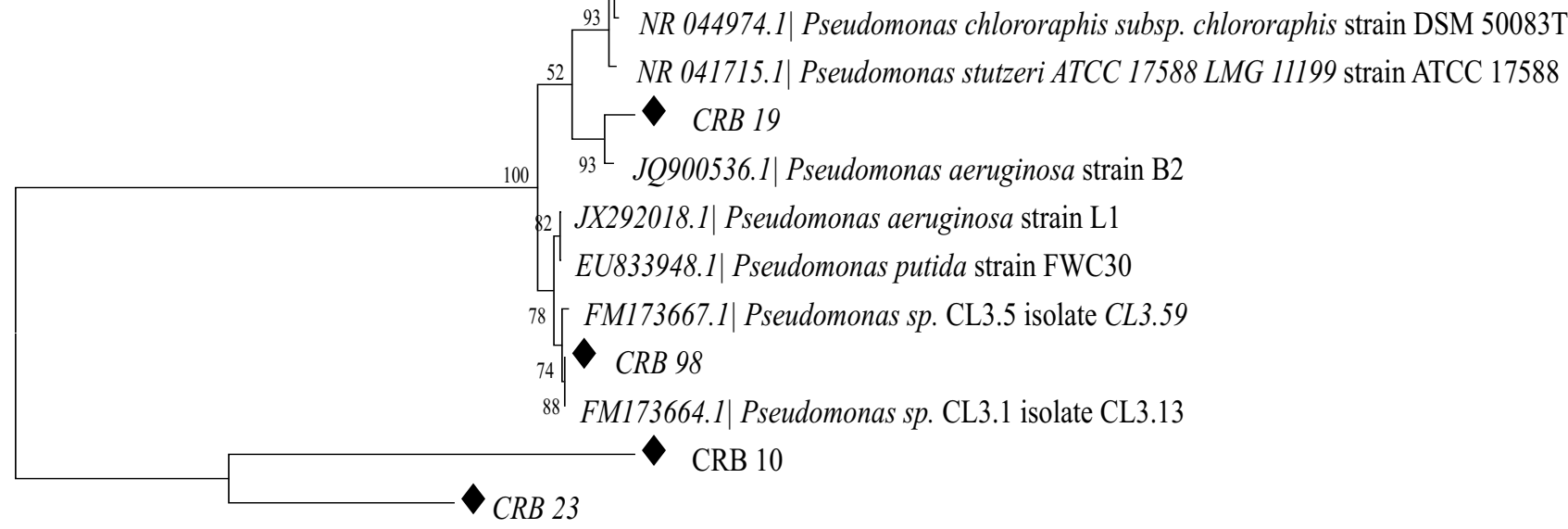

0.1

Fig 2 Phylogenetic tree based on 16S rRNA gene of Bacillus sp. (A) and Pseudomonas sp. (B) compared with 16S rRNA gene of other species. Scale showed that distance evolution on the branch length, while the numbers on the branches indicate bootstrap values.

stop the growth.

Bacteria PGPR had ability in producing IAA and insert that into pool auxin hormone of plant. Plant roots were the most sensitive organ to fluctuations of IAA levels and its response of increase exogenous IAA widespread in the number of primary root elongation, 
lateral root formation and adventitious roots until termination of growth on the plant (Leveau and Lindow 2005). Patten and Glick (2002) also stated that the high of exogenous IAA would increase ethylene production which would effect to inhibition of roots growth. Other mechanism that had capable to support as plant promoting growth by $\mathrm{CR}$ and $\mathrm{CRB}$ are their ability in phosphate solubilizing which one of the essential nutrients for plants. Phosphate in soils were present in bound form. Phosphate bound with aluminium $\left(\mathrm{Al}^{3+}\right)$, iron $\left(\mathrm{Fe}^{3+}\right)$, calcium $\left(\mathrm{Ca}^{2+}\right)$, and magnesium $\left(\mathrm{Mg}^{2+}\right)$. Thus, only it a small fraction which could be absorbed by plants (Trivedi and Pandey, 2007), PGPR had been ability to solubilyze bound phosphate so that could be absorbed by plants.

Screening of drought tolerant aimed to determine of isolates which could survive on drought condition so their can survive when applied in the field. The addition of PEG 6000 on medium which bound water molecule were simulated as drought stress so that could reduce of potential water value. Consequently, reduction of potential water value by the high of PEG concentration on medium would cause decreasing survival of bacterial population. Optical density values linearly related to water potential contained on medium. Reduction of water potential values would decrease number of bacterial population. Therefore, PEG 6000 which soluble in water used to reduce of water potential value. Water potential associated with level of drought would affect to capability survive of bacteria. Isolates that had cappability to grow on medium with a certain of potential water value and OD value $\geq 0.4$ classified as drought tolerant isolates (Alikhani and Mohamadi 2010).

Majority, the result of antagonism test for plant promoting growth and drought tolerant isolates showed the same result for isolate $\mathrm{CR}$ and $\mathrm{CRB}$ were used as the test culture and targets culture, respectively. Formation of clearing zone around paper disc occured by production of certain compounds by bacteria test that inhibited growth of bacterial target or while back of test. Isolates with different results founded in isolates CR 33 which wasn't antagonis to CRB 98 when CR 33 was used as target isolates and isolate CR 83 wasn't antagonis to CRB 19 when CR 83 as a target. This result might be caused by differences substance when isolates were used as targets and test isolates. When isolate used as targets, the substances were intact cells while tested back were used supernatant.

Tolerances to the drought stress were characterized by production of EPS. This indicates that production of EPS linearly with stress experienced by cells as a form of physiological adaptation so that cells could survive. Therefore, the capability in producing EPS by bacterial cells used as drought tolerance criteria for bacteria (Sandhya et al. 2009). Production of EPS would increased by cells during stress experienced as a form of physiological adaptation so that cells could survive. EPS were produced to protect bacterial cells from drought, heavy metals or other environmental stresses, including host immune response and to produce a biofilm which could increase survival cell in specific ecological niches (Ozturk and Aslim 2010). Quantity and composition of EPS vary greatly depending on genus and species of bacteria, in some cases dependent on environmental conditions for growth. In addition, the carbon sources in medium was functioning as a component of cell formation, source of energy required for synthesis and EPS excretion (Santi 2011).

B. subtilis could produce levan exopolysaccharide within a range $0.32-86.3 \mathrm{~g} \mathrm{~L}^{-1}$ using sucrose as substrate. A total of 16 genes of operon EPS ( $y v e K y v f F$ ) involved in the biosynthesis, modification and expenditure. Recently, two genes eps $\mathrm{G}$ (yveQ) and eps $\mathrm{H}(y v e R)$ had been identified that might be involved in the biosynthesis. eps $\mathrm{G}$ encodes a protein that might be involved in EPS polymerization and eps $\mathrm{H}$ encodes a glycosyltransferase (Marvasi et al. 2010). Species of Pseudomonas has been known for their capability producing EPS in alginate type. Approximately 24 genes in $P$. aeruginosa were identified. Cluster consisted of 12 structural genes (alg D, alg8, alg44, $\operatorname{alg} \mathrm{K}, \operatorname{alg} \mathrm{e}, \operatorname{alg} \mathrm{G}, \operatorname{alg} \mathrm{X}, \operatorname{alg} \mathrm{L}, \operatorname{algl}, \operatorname{alg} \mathrm{J}, \operatorname{alg} \mathrm{F}$, and algae) were clustered in a single operon approximately $3.96 \mathrm{Mb}$. Only algC genes located on separate chromosomes. Those gene encodes for phosphomannomutase involved in rhamnolipid and lipopolysaccharide biosynthesis. Operon was containing all the genes that encode proteins involved in the biosynthesis of alginate ( $\operatorname{alg\mathrm {D}}$ and algae for precursor synthesis, algl, $\operatorname{alg\mathrm {J}}$, and $\operatorname{alg} \mathrm{F}$ for acetylation, $\operatorname{alg} \mathrm{G}$ to epimerisasi, algL for degradation) (Hay et al. 2010).

The potent isolates CRB 19 and CR 90 exhibited the highest activity of EPS secretion and based on $16 \mathrm{~S}$ rRNA analysis revealed CRB 19 and CR 90 belonged to P. aeruginosa strain $\mathrm{B} 2$ and Brevibacillus brevis $\mathrm{B} 33$, respectively. Other studies showed that those group was known as promoting growth in plants. P. aeruginosa FP6 isolated from rhizosphere had been known for their capability as phosphate solubilizing, producer of IAA hormone, ammonia, sidherophore, and cells wall 
degrading enzyme such as cellulase, chitinase, and protease. Inoculation of cowpea (Vigna unguiculata) seeds by those bacteria given significantly effect $(\mathrm{P}<0.05)$ for enhanced seed germination $(92 \%)$, seedling vigor index, plant height, and also fresh and dry weight in compared with control (Bhakthavatchalu et al. 2013). B. brevis strain IPC11 also known could increase seed germination, producing phenylalanine ammonia lyase enzyme and reduced cancer disease in tomato (Girish and Umesha 2005). Finally, the potent isolates of Bacillus sp. CR and Pseudomonas sp. CRB have capability to live in drought condition and could be developed as inoculants in dry land agriculture when it put in medium carrier as biofertilizer.

\section{ACKNOWLEDGMENTS}

The research was supported by I-MHERE B2c. Institut Pertanian Bogor (IPB) and a grant from the Collaborative Research Project of KKP3N, Department of Agriculture, Indonesia to ATW. Therefore, authors thank and appreciate for all the supports given to carry out this research.

\section{REFERENCES}

Alikhani HA, Mohamadi L. 2010. Assesing tolerance of rhizobial lentil symbiosis isolates to salinity and drought in dry land farming condition. $19^{\text {th }}$ World Congress of Soil Science, Soil Solutions for Changing World. Australia, 1-6 August 2010.

Bhakthavatchalu S, Shivakumar S, Sullia SB. 2013. Characterization of multiple plant growth promotion traits of Pseudomonas aeruginosa FP6, a potential stress tolerant biocontrol agent. Ann Biol Res. 4(2):214-223.

Dey, Pal KK, Bhatt DM, Chauhan SM. 2004. Growth promotion and yield enhancement of peanut (Arachis hypogaea L.) by application of plant growth promoting rhizobacteria. Microbiol Res. 159(4):371-394. doi: 10.1016/j.micres.2004.08.004.

Donot, Fontana, Baccoua, Schorr-Galindo. 2011. Microbial exopolysaccharides: main examples of synthesis, excretion, genetics and extraction. Rev Carbohyd Polysac. 87(2):951-962. doi:10.1016/j.carbpol.2011.0 8.083.

Dubois M, Gilles KA, Hamilton JK, Rebers PA, Smith F. 1956. Colorimetric methods for determination of sugars of related substances. Anal Chem. 28(3):350356. doi:10.1021/ac60111a017.

Girish N, Umesha S. 2005. Effect of plant growth promoting rhizobacteria on bacterial cancer of tomato. Arch Phytopatol Plant Protect. 38(3):235-243.

Hay ID, Ur Rehman Z, Ghafoor A, Rehm BHA. 2010. Bacterial biosynthesis of alginates. Chem Technol
Biotechnol. 85(6):752-759. doi:10.1002/jctb.2372.

Husen E, Wahyudi AT, Suwanto A, Giyanto. 2011. Growth enhancement and disease reduction of soybean by 1aminocyclopropane-1-carboxylate deaminase-producing Pseudomonas. Am J Appl Sci. 8(11):1073-1080.

Kaci Y, Heyraud A, Barakat M, Heulin T. 2005. Isolation and identification of an EPS-producing Rhizobium strain from arid soil (Algeria) : characterization of its EPS and the effect of inoculation on wheat rhizosphere soil structure. Res Microbiol. 156(4):522-531.doi:10.1002/ jctb. 2372 .

Leveau JHJ, Lindow SE. 2005. Utilization of the plant hormone indole-3-acetic acid for growth by Pseudomonas putida strain 1290. Appl Environ Microbiol. 71(5):2365-2371. doi :10.1128/AEM.71.5.2 365-2371.2005.

Marvasi M, Visscher PT, Martinez LC. 2010. Exopolymeric substances (EPS) from Bacillus subtilis : Polymers and genes encoding their synthesis. FEMS Microbiol Lett. 313(1):1-9. doi:10.1111/j.1574-6968.2010.02085.x.

Michel BE, Kauffman MR. 1973. The osmotic potential of polyethylene glycol 6000. Plant Physiol. 51(5):914916.

Marchesi JR, Sato T, Weightman AJ, Martin TA, Fry JC, et al. 1998. Design and evaluation of useful bacterium specific PCR primers that amplify genes coding for bacterial 16S-rRNA. Appl Environ Microbiol. 64(2):795-799.

Ozturk S, Aslim B. 2010. Modification of exopolysaccharide composition and production by three cyanobacterial isolates under salt stress. Environ Sci Pollut Res. 17(3):595-602.

Patten CL, Glick BR. 2002. Role of Pseudomonas putida indoleacetic acid in development of the host plant root system. Appl Environ Microbiol. 68(8):3795-3801. doi:10.1128/AEM.68.8.3795-3801.2002.

Trivedi P, Pandey A. 2007. Low temperature phosphate solubilization and plant growth promotion by psychrotrophic bacteria, isolated from Indian Himalayan region. Microbiol. 2(5):454-461.

Sandhya, Ali SKZ, Minakshi G, Gopal R, Venkateswarlu. 2009. Alleviation of drought stress effects in sunflower seedlings by the exopolysaccharides producing Pseudomonas putida strain GAP-P45. Biol Fertil Soils. 46(1):17-26. doi:10.1007/s00374-009-0401-z.

Santi LP. 2011. The Role of exopolysaccharide producing bacteria in aggregation of a sandy soil texture [dissertation]. Bogor(ID) : Institut Pertanian Bogor.

Somasegaran P, Hoben HJ. 1985. Methods in LegumeRhizobium Technology. Hawaii:Department of Agronomy and Soil Science, University of Hawaii.

Wahyudi AT, Astuti RP, Widyawati A, Meryandini A, Nawangsih AA. 2011a. Characterization of Bacillus sp. strains isolated from rhizosphere of soybean plants for their use as potential plant growth for promoting rhizobacteria. J Microbiol Antimicrob. 3(2):34-40.

Wahyudi AT, Astuti RI, Giyanto. 2011b. Screening of Pseudomonas sp. isolated from rhizosphere of soybean 
plant as plant growth promoter and biocontrol agent. Am J Agric Biol Sci. 6:134-141.

Wibowo ST. 2008. Hormone IAA content, nutrient uptake, and growth of some cultivated crops in response to the application of biofertilizer [thesis]. Bogor (ID) : Institut Pertanian Bogor.
Zhao Y, Christensen SK, Fankhauser C, Cashman JR, Johen JD, Weigel D, Chory J. 2001. A role for flavin monooxygenase like enzyme in auxin biosynthesis. Science 291(5502):306-309. 